\title{
Assessment of Gas-Surface Interaction Models in DSMC Analysis of Rarefied Hypersonic Flow
}

\author{
Jose F. Padilla* and Iain D. Boyd ${ }^{\dagger}$ \\ University of Michigan, Ann Arbor, MI, 48109
}

\begin{abstract}
The aerothermodynamics of spacecraft entering a planetary atmosphere are sensitive to the level of gas-surface accommodation governed by the gas-surface interaction. The modeling of this interaction plays an integral role in the solid surface boundary condition of the Direct Simulation Monte Carlo (DSMC) method. The Maxwell, and Cercignani, Lampis and Lord (CLL) gas-surface interaction models are examined. Existing windtunnel test results of rarefied hypersonic flow over flat surfaces enable the assessment of these gassurface interaction models for DSMC simulations for this kind of flow condition. These models gave the same boundary layer velocity profiles at $50 \%$ to full gas-surface accommodation. Approximately, $90 \%$ gas-surface accommodation yielded the overall best agreement between the simulations and windtunnel data, reported by Cecil and McDaniel [AIAA Paper 2005-4695]. Regarding molecular velocity distributions next to the surface, the gas-surface interaction models result in similar horizontal component distributions, but distinct vertical component distributions. Molecular velocity distributions also reveal translational nonequilibrium very near the surface due to surface reflected molecules, within 5 local mean-free-paths above the surface. Within a region of significant translational nonequilibrium, the distributions are better characterized by the most probable value, rather than the mean value. Regarding scattering distributions, the Maxwell model results in distributions with unrealistic peaks due to specular reflection; however, the CLL model results in petal-shaped distributions, similar to observations of molecular beam studies. Moreover, while the Maxwell scattering distributions experienced abrupt changes with increasing accommodation and position, the CLL distributions varied smoothly. Nevertheless, both yield good agreement with the PLIF windtunnel test boundary layer velocity profiles using a proper specification of gas-surface accommodation.
\end{abstract}

\section{Nomenclature}

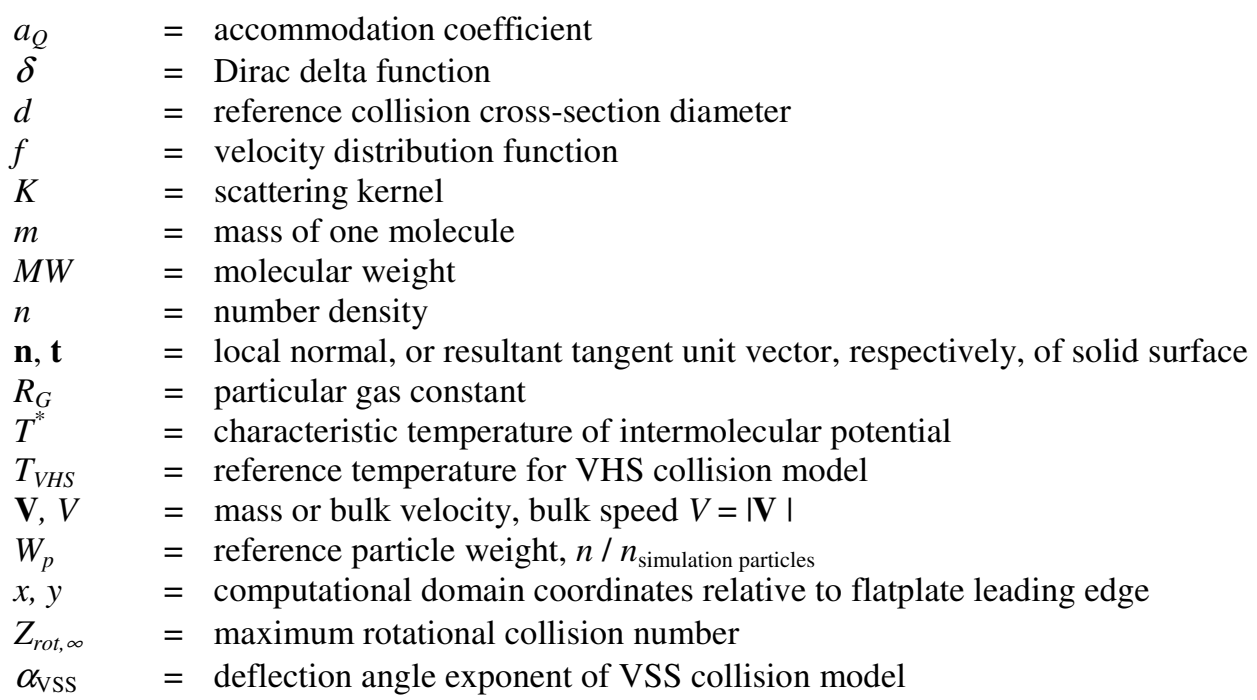

\footnotetext{
*Graduate Student, Department of Aerospace Engineering, AIAA Student Member

${ }^{\dagger}$ Professor, Department of Aerospace Engineering, AIAA Associate Fellow
} 


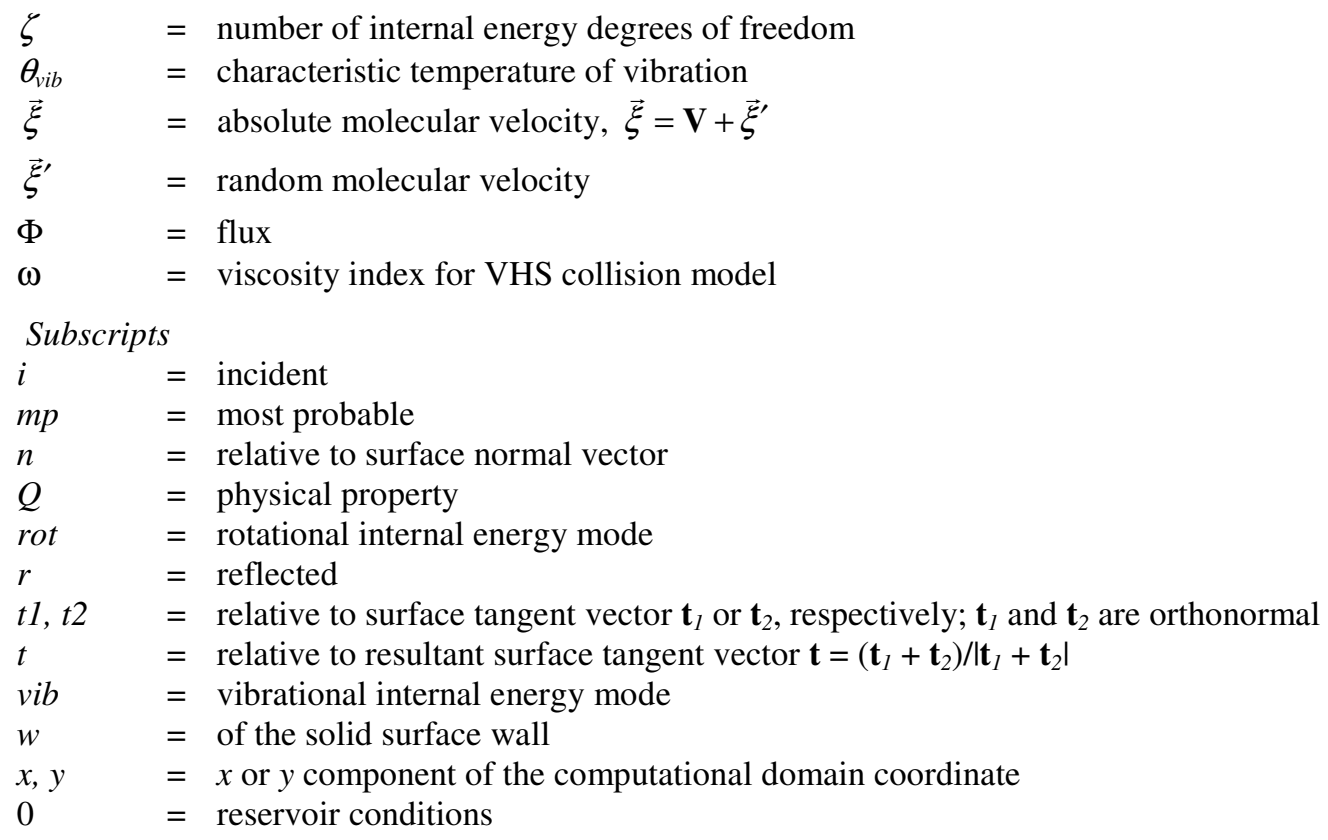

\section{Introduction}

Human space flight continues to require careful consideration of atmospheric entry. Designing atmospheric entry vehicles involves analyzing the aerothermodynamics of hypersonic flow conditions through a spectrum of Knudsen numbers from rarefied to continuum. This study focuses on rarefied flow conditions, at a level of rarefaction commensurate to the initial phase of spacecraft entry into Earth's atmosphere. For the rarefied flow conditions of interest, the analysis must be made in terms of kinetic theory, where molecular collisions play a significant role, in order to correctly predict the respective aerodynamics and stability. Aerothermodynamics is governed by the momentum and energy transferred from the incoming gas flow to the surface of a vehicle. These transfers are governed by the collisions between the gas molecules and the solid surface. The collision process between a gas molecule and a solid surface is termed a gas-surface interaction. In kinetic theory, the gas-surface interaction forms a boundary condition between the gas molecules and the solid surface. For scales relevant to kinetic theory, the gassurface interactions are modeled with parameters having macroscopic character, in order to have manageable and efficient computations. The smallest geometric spatial scale considered in this study is on the order of $0.1 \mathrm{~mm}$, based on the minimum computational mesh cell size. Although various gas-surface interaction models have been proposed over the past century and a half, the validity of these models remains tenuous for hypersonic rarefied flow conditions. This study employs numerical simulation, using the Direct Simulation Monte Carlo (DSMC) method, to scrutinize two of the most common gas-surface interaction models used with DSMC: the Maxwell model, and the Cercignani, Lampis and Lord (CLL) model. In the literature, comparisons of flow field properties between DSMC and laboratory data of rarefied hypersonic flow are uncommon. Hence, this study also intends to generate new comparisons of this kind. In particular, the study compares shock or boundary layer properties of rarefied hypersonic flow near the leading edge of a flat plate between DSMC and existing windtunnel data. These comparisons involve the assessment of the prediction capabilities of the gas-surface interaction models through parametric analysis of boundary layer velocity profiles. In addition, the study examines probability distribution plots of the molecular velocity components at specified points in the flow field, and of molecular angle of reflection at specified points on the solid surface. The study is presented in the following order: first, the underlying theory is presented; second, the DSMC implementation is explained; third, the windtunnel test simulations are discussed; and finally, the results are summarized and future directions are suggested. 


\section{Gas-Surface Interaction Models}

\section{A. Modeling Concepts}

\section{Interaction Parameters}

Parameters employed in gas-surface interaction models are called interaction parameters. For larger than the nanometer scale, these parameters are relevant to a macroscopic description; typically, they are accommodation coefficients. An accommodation coefficient is a numerical description about the degree to which a flow of gas accommodates kinetically or thermally with a solid surface, while interacting with the surface. The accommodation coefficient of molecular property $Q$ is defined in terms of incident and reflected fluxes as follows: ${ }^{1,2}$

$$
a_{Q}=\frac{\Phi_{i}^{Q}-\Phi_{r}^{Q}}{\Phi_{i}^{Q}-\Phi_{w}^{Q}}
$$

where $\Phi_{i}^{Q}$ and $\Phi_{r}^{Q}$ are the incident and reflected fluxes of $Q$, respectively, and $\Phi_{w}^{Q}$ is the reflected flux of $Q$ corresponding to full accommodation. Common examples of $Q$ are the total energy $E$, normal momentum $m \xi_{n}$, and tangential momentum $m \xi_{t}$. The total energy, normal momentum and tangential momentum accommodation coefficients are defined, with the usual notation, by $\alpha \equiv a_{E}, \sigma_{n} \equiv a_{m \xi_{n}}$, and $\sigma_{t} \equiv a_{m \xi_{t}}$, respectively.

\section{Scattering Kernel}

In kinetic theory analysis, the gas-surface interaction model is used as a boundary condition. A formal mathematical construct for a gas-surface interaction model is the scattering kernel formulation. ${ }^{3}$ A scattering kernel $K\left(\vec{\xi}_{i}, \vec{\xi}_{r}\right)$ represents the probability density that an incident molecule with velocity $\vec{\xi}_{i}$ is reflected with velocity $\vec{\xi}_{r}$ at essentially the same time and place. It bridges the velocity distribution functions, $f_{i}\left(\vec{\xi}_{i}\right)$ and $f_{r}\left(\vec{\xi}_{r}\right)$, of the incident and reflected molecules, respectively, through the following integral transform:

$$
\xi_{n, r} f_{r}\left(\vec{\xi}_{r}\right)=\int_{\xi_{n, i}<0}\left|\xi_{n, i}\right| f_{i}\left(\vec{\xi}_{i}\right) K\left(\vec{\xi}_{i}, \vec{\xi}_{r}\right) d \vec{\xi}_{i}
$$

where each gas-surface interaction is independent of others and the average interaction time is small relative to the temporal evolution of $f$. In addition, the scattering kernel satisfies the following three criteria: positivity, normalization and reciprocity. These criteria are expressed mathematically as follows:

$$
\begin{gathered}
K\left(\vec{\xi}_{i}, \vec{\xi}_{r}\right) \geq 0 \\
\int_{\xi_{n, r}>0} K\left(\vec{\xi}_{i}, \vec{\xi}_{r}\right) d \vec{\xi}_{r}=1 \\
\left|\xi_{n, i}\right| f_{M}\left(\vec{\xi}_{i}\right) K\left(\vec{\xi}_{i}, \vec{\xi}_{r}\right)=\left|\xi_{n, r}\right| f_{M}\left(\vec{\xi}_{r}\right) K\left(-\vec{\xi}_{i},-\vec{\xi}_{r}\right)
\end{gathered}
$$

where $f_{M}$ is the Maxwellian velocity distribution in equilibrium with the solid surface:

$$
f_{M}(\vec{\xi}) d \vec{\xi}=\left(2 \pi R_{G} T_{w}\right)^{-\frac{3}{2}} \exp \left(-\frac{\xi^{2}}{2 R_{G} T_{w}}\right) d \vec{\xi}
$$

The product $f(\vec{\xi}) d \vec{\xi}$ is the probability of any given molecule to have velocity $\vec{\xi}$ within the range $d \vec{\xi}$. The reciprocity condition, ${ }^{4,5}$ Eqn. (5), is the equilibrium condition for gas-surface interactions. It must be satisfied when the flow is in equilibrium with the solid surface. 


\section{B. Models}

\section{Maxwell Model}

Historically, the first gas-surface interaction model for kinetic theory is the model developed by Maxwell. ${ }^{6}$ It considers two kinds of interactions: the specular and diffuse interactions. A specular interaction or reflection occurs when an incident molecule collides with the molecular structure of a solid surface in such a way that it rebounds elastically as if hitting a flat surface. This type of collision occurs when the gas molecule collides with a peak of the solid surface molecular structure, assuming the gas and solid molecules are rigid elastic spheres. The collision results in an inversion of the surface normal component of the molecule's velocity and no change in its tangential component. Thus, the angle of reflection is the same as the angle of incidence. Meanwhile, the molecule's thermal energy is assumed to remain the same. A diffuse interaction occurs when an incident molecule interacts with the molecular structure of the solid surface in such a way that it attains thermal equilibrium with the surface and then rebounds from the surface according to the Maxwellian velocity distribution at the local surface temperature. The Maxwell model considers a fraction $a_{M}$ of the incident molecules to be temporarily absorbed by the surface and then reflected diffusely from the surface; the remaining incident molecules are assumed to reflect specularly. Hence, Maxwell's fraction $a_{M}$ expresses the probability of a diffuse reflection. The scattering kernel for this model is written as follows: ${ }^{7}$

$$
K_{M}\left(\vec{\xi}_{i}, \vec{\xi}_{r}\right)=\left(1-a_{M}\right) \delta\left(\vec{\xi}_{i}-\vec{\xi}_{r, \text { specular }}\right)+a_{M} f_{M}\left(\vec{\xi}_{r}\right)\left|\vec{\xi}_{r} \cdot \mathbf{n}\right|
$$

where $\vec{\xi}_{r, \text { specular }}$ is the molecular velocity of specular reflection.

\section{Cercignani, Lampis, and Lord Model}

The scattering distribution predicted by the Maxwell model, for a beam of molecules targeted onto a surface at a specified angle of incidence, has a circular shape due to the diffuse reflections combined with a protruding peak due to the specular reflections. If the molecules in the beam are all traveling at the same velocity, then the peak is a line at the specular angle of reflection. However, contrary to the expected distributions given by the Maxwell model, ${ }^{8,9}$ molecular beam experiments have shown scattering distributions to be petal-shaped. Because of these findings, various models have been developed to match the observed scattering distributions. ${ }^{10,11,12,13,14}$ The best of these models is the Cercignani and Lampis $(\mathrm{CL})$ mode $^{13}$ because it involves well defined interaction parameters, parameters that can be expressed in the form of Eqn. (1), and it involves a well defined mathematical framework, namely, the scattering kernel construction. The CL model interaction parameters are the accommodation coefficient for the tangential momentum $\sigma_{\mathrm{t}} \equiv a_{Q}$, where $Q=m \vec{\xi} \cdot \mathbf{t}$, and the accommodation coefficient for the normal part of the kinetic energy $\alpha_{n} \equiv a_{Q}$, where $Q=\frac{1}{2} m(\vec{\xi} \cdot \mathbf{n})^{2}$. The scattering kernel has the following form: ${ }^{3,15,16}$

$$
\begin{array}{r}
K_{C L}\left(\vec{\xi}_{i}, \vec{\xi}_{r}\right)=\frac{\left[\alpha_{n} \sigma_{t}\left(2-\sigma_{t}\right)\right]^{-1}}{2 \pi\left(R_{G} T_{w}^{2}\right)} \xi_{n, r} \exp \left(-\frac{\xi_{n, r}^{2}+\left(1-\alpha_{n}\right) \xi_{n, i}^{2}}{2 \alpha_{n} R_{G} T_{w}}-\frac{\left[\xi_{t, r}-\left(1-\sigma_{t}\right) \vec{\xi}_{t, i}\right]^{2}}{2 \sigma_{t}\left(2-\sigma_{t}\right) R_{G} T_{w}}\right) \\
\times I_{0}\left(\frac{\sqrt{1-\alpha_{n}} \xi_{n, r} \xi_{n, i}}{\alpha_{n} R_{G} T_{w}}\right) \quad\left(\xi_{n, i}<0 ; \xi_{n, r}>0 ; 0 \leq \sigma_{t} \leq 2 ; 0 \leq \alpha_{n} \leq 1\right)
\end{array}
$$

where $I_{0}$ is the modified Bessel function of the first kind and of zeroth order, and $\vec{\xi}_{t}$ is the sum of the tangential components of velocity.

The CL model is implemented into DSMC code by a simple algorithm, with a level of complexity not much greater than the implementation of the Maxwell model. Lord originally made the transformation to this algorithm with the help of a graphical representation of the CL model. ${ }^{17}$ This algorithm forms the basic Cercignani, Lampis, and Lord (CLL) model. Table 1 presents the algorithm. In these equations, $\alpha_{\mathrm{t}} \equiv a_{Q}$, with $Q=\frac{1}{2} m\left(\vec{\xi}_{\cdot} \cdot \mathbf{t}_{i}\right)^{2}$, where $\mathbf{t}_{i}$ is either of the surface tangent vectors. In other words, $\alpha_{\mathrm{t}}$ is the accommodation coefficient for the part of the kinetic energy along either tangent to the surface; it is assumed independent of direction along the surface. 
Table 1. Algorithm equations of the CLL model: reflected molecular velocity components relative to local surface unit vectors

\begin{tabular}{lll}
\hline Normal Component & Tangent 1 Component & Tangent 2 Component \\
$r_{1}=\sqrt{-\alpha_{n} \ln x_{1}}$ & $r_{3}=\sqrt{-\alpha_{t} \ln x_{3}}$ & $r_{5}=\sqrt{-\alpha_{t} \ln x_{5}}$ \\
$\theta_{2}=2 \pi x_{2}$ & $\theta_{4}=2 \pi x_{4}$ & $\theta_{6}=2 \pi x_{6}$ \\
$\xi_{n, m}=\left|\xi_{n, i} / \xi_{m p, w}^{\prime}\right| \sqrt{1-\alpha_{n}}$ & $\xi_{t, m}=\left|\xi_{t 1, i} / \xi_{m p, w}^{\prime}\right| \sqrt{1-\alpha_{t}}$ & $\xi_{t 2, r}=\xi_{m p, w}^{\prime} r_{5} \cos \theta_{6}$ \\
$\xi_{n, r}=\xi_{m p, w}^{\prime} \sqrt{r_{1}^{2}+\xi_{n, m}^{2}+2 r_{1} \xi_{n, m} \cos \theta_{2}}$ & $\xi_{t 1, r}=\xi_{m p, w}^{\prime}\left(\xi_{t, m}+r_{3} \cos \theta_{4}\right)$ &
\end{tabular}

Auxiliary: $\quad x_{i}$ are random numbers uniformly distributed between 0 and $1 ; \quad \xi_{m p, w}^{\prime}=\sqrt{2 R_{G} T_{w}} ; \quad \alpha_{t}=\sigma_{t}\left(2-\sigma_{t}\right)$

\section{Implementation of the DSMC Method}

The standard governing equation of kinetic theory is the Boltzmann equation. In its general form, it is a nonlinear integro-differential equation in terms of a function describing the distribution of molecule velocities, the velocity distribution function. Hence, the Boltzmann equation is solved numerically in practical applications. Various numerical methods for its solution are proposed in the literature. In fact, with the rise of the modern computer, a statistical simulation method was developed to simulate rarefied gas flows without direct reference to the Boltzmann equation, although it uses the same physical concepts and provides an indirect solution. The Direct Simulation Monte Carlo (DSMC) method $^{18}$ tracks a representative system of simulation molecules through a computational domain while simulating collisions stochastically. In addition, it is actually more general than the Boltzmann equation because it can handle collisions with more than two molecules at one time. This study employs a general, object-oriented, cell-based implementation of the DSMC method called MONACO. ${ }^{19}$ MONACO can be executed on serial or parallel computer systems, and it can handle structured or unstructured grids, with two or three spatial dimensions. It employs various procedures for handling collisions. These deal with gaseous intermolecular collisions and gas-surface interactions. For gaseous intermolecular collisions, an intermolecular force model, described by a molecular shape, help compute the collision dynamics. MONACO provides the option of using either the variable hard sphere (VHS) ${ }^{20}$ model or variable soft sphere (VSS) ${ }^{21}$ model. In addition, for internal energy exchange, it uses the variable rotational energy exchange probability model developed by Boyd, ${ }^{22}$ and, when vibrational energy exchange is relevant, it uses the variable vibrational energy exchange probability model developed by Vijayakumar, et al. ${ }^{23}$ For the gas-surface interactions, MONACO originally used the Maxwell model. For this study, the option of using either the Maxwell or the CLL gas-surface interaction model is added. In addition, procedures are added to extract probability distributions of velocity at requested points in space or of reflected velocity at requested points on a solid surface.

The implementation of the Maxwell and CLL gas-surface interaction models includes an internal energy accommodation coefficient, $a_{E_{-} i n t}$. For the Maxwell model, the total internal energy accommodation coefficient is set equal to Maxwell's fraction, $a_{E_{-} \text {int }}=a_{M}$. For the CLL model, it is independently specified, as a third accommodation coefficient. For both models, the internal energy accommodation coefficient indicates the probability that the molecular internal energy will fully accommodate to the solid surface or wall temperature; otherwise, the internal energy is unchanged. Consideration is given to the rotational and vibrational modes of internal energy.

\section{Windtunnel Test Simulations}

\section{A. General Description}

To examine the gas-surface interaction models, this research involves computer simulations of existing windtunnel tests examining rarefied hypersonic flow over a flat surface or plate. The windtunnel tests, reported by Cecil and McDaniel, ${ }^{24}$ in 2005, uses planar laser induced fluorescence (PLIF) of seeded iodine, within a free jet expansion of nitrogen, to measure boundary layer flow properties. Details of the windtunnel test section apparatus, including the geometry of the flat plate windtunnel model, is given in Ref. 24. 
The computer simulations of the flow-field are performed assuming two-dimensional flow. In addition, they use rectangular spatial domains that cover a region near the leading edge of the flat plate, which includes an adequate number of windtunnel test measurement locations. Each domain is divided into quadrilateral cells to form twodimensional computational meshes. The cells have sizes on the order of the local mean-free-path, according to the DSMC constraint. Throughout each mesh, a uniform particle weight $W_{p}$ is assigned. The meshes are generated with HyperMesh $^{25}$ and converted to MONACO readable format with an in-house grid-conversion code.

To simulate Cecil and McDaniel's experiment, pure nitrogen is assumed. The corresponding input parameters are listed in Table 2. The inflow properties are shown in Figs. 1 (a) and (b). The inflow velocity profiles are provided by Cecil and McDaniel. The translational temperature and number density inflow profiles are calculated from the inflow velocity profiles and the windtunnel test reservoir conditions, $T_{0}=300 \mathrm{~K}$ and $p_{0}=1.79 \mathrm{~atm}$. This calculation assumes that the reservoir contains an ideal gas and that it is expelled isentropically as a free jet expansion. The Knudsen number, based on the average inflow local mean-free-path and the flat plate length, is about 0.005 , within the rarefied transitional regime.

Table 2. Physical input parameters for pure nitrogen MONACO simulation of PLIF windtunnel test

\begin{tabular}{ll|ll}
\hline Species Data & & \multicolumn{2}{|l}{ Collision Cross-Section Data } \\
\hline Species & $\mathrm{N}_{2}$ & $T_{V H S}$ & $290 \mathrm{~K}$ \\
$M W$ & 28.01 & $\omega$ & 0.7 \\
$\zeta_{\text {rot }}$ & 2.0 & $d$ & $4.11 \times 10^{-10} \mathrm{~m}$ \\
$\zeta_{\text {vib }}$ & 0.0 & $\alpha_{\mathrm{VSS}}$ & 1.784 \\
$\theta_{\text {vib }}$ & $3390 \mathrm{~K}$ & & \\
$T^{*}$ & $91.5 \mathrm{~K}$ & \multicolumn{2}{|l}{ Solid Surface Data } \\
\cline { 2 - 3 }$Z_{\text {rot } \infty}$ & 18.1 & $T_{\mathrm{w}}$ & $300 \mathrm{~K}$ \\
\hline
\end{tabular}

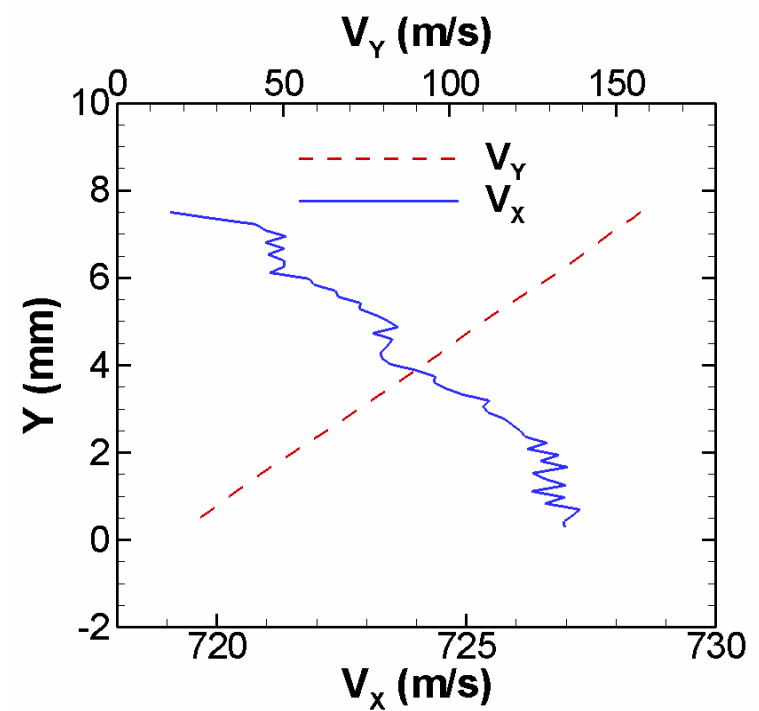

(a) Velocity Profiles

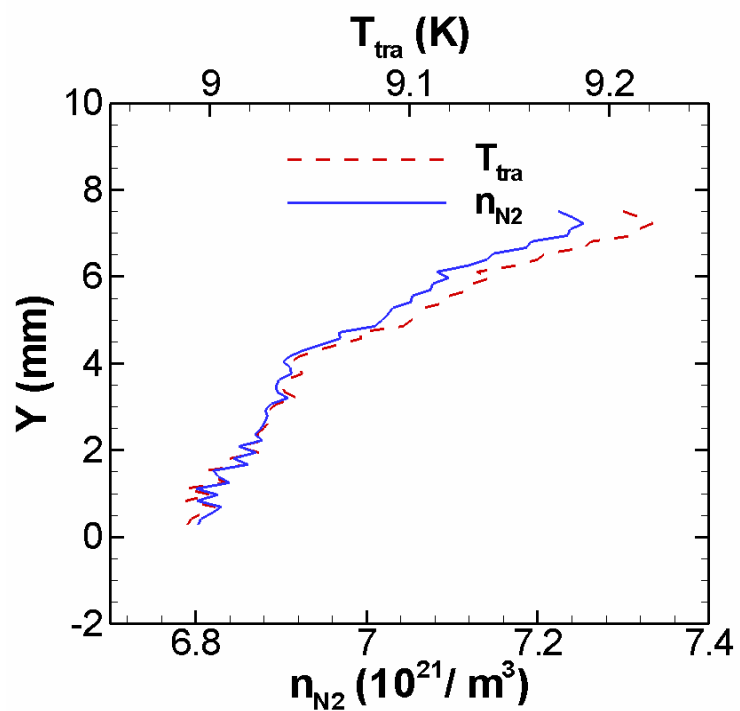

(b) Number Density and Temperature Profiles

Figure 1. Nonuniform inflow conditions for MONACO simulating PLIF windtunnel test

Using these flow conditions, simulations are first made with the Maxwell gas-surface interaction model, at various values of Maxwell's fraction, $a_{M}=0,0.25,0.50,0.75$, and 1.00. Then, simulations are made with the CLL gas-surface interaction model, at various values of tangential momentum accommodation, $\sigma_{t}=0,0.25,0.50,0.75$, and 1.00, with full normal kinetic energy accommodation, $\alpha_{n}=1.00$. In addition, the sensitivity to normal kinetic energy accommodation is examined with similar levels of accommodation, $\alpha_{n}=0,0.25,0.50,0.75$, and 1.00, while maintaining zero tangential momentum accommodation, $\sigma_{t}=0$. Each of the simulations with the CLL model assumes full internel energy accommodation, $a_{E_{-} i n t}=1$. All of the pure nitrogen simulations require computational parameters similar to those summarized in Table 3. 
Table 3. Typical computational properties of a pure $\mathbf{N}_{2}$ simulation of the PLIF windtunnel test

\begin{tabular}{ll|ll}
\hline$W_{p}$ & $1.0 \times 10^{12}$ & Time-step size & $1.0 \times 10^{-8} \mathrm{~s}$ \\
Particles & $1,915,000$ & Time-steps & $75,000(45,000$ sampled $)$ \\
Cells & 19,067 & Simulation Real Time & $\sim 3.5 \mathrm{hr}$ \\
Processors & 4 & & \\
\hline "1.4GHz AMD Opteron 240 or 28GHz AMD Opteron 254, & & \\
\hline
\end{tabular}

1.4GHz AMD Opteron 240 or 2.8GHz AMD Opteron 254, 1GB RAM capacity per processor

\section{B. Simulation Results and Discussion}

\section{Contour Plots}

The variation of Maxwell's fraction $a_{M}$ from fully specular to fully diffuse results in a variation in boundary layer size from non-existent to maximum extent. Part of this variation is illustrated by the half and fully diffuse cases in Figs. 2 (a) and (b). These figures also illustrate the nonuniform inflow boundary through streamlines of bulk flow speed $V$. A similar variation in boundary layer size is given by the parametric analysis with the CLL model involving the variation in tangential momentum accommodation $\sigma_{t}$, with full normal kinetic energy accommodation $\alpha_{n}=1$, except that the boundary layer doesn't completely disappear at $\sigma_{t}=0$.
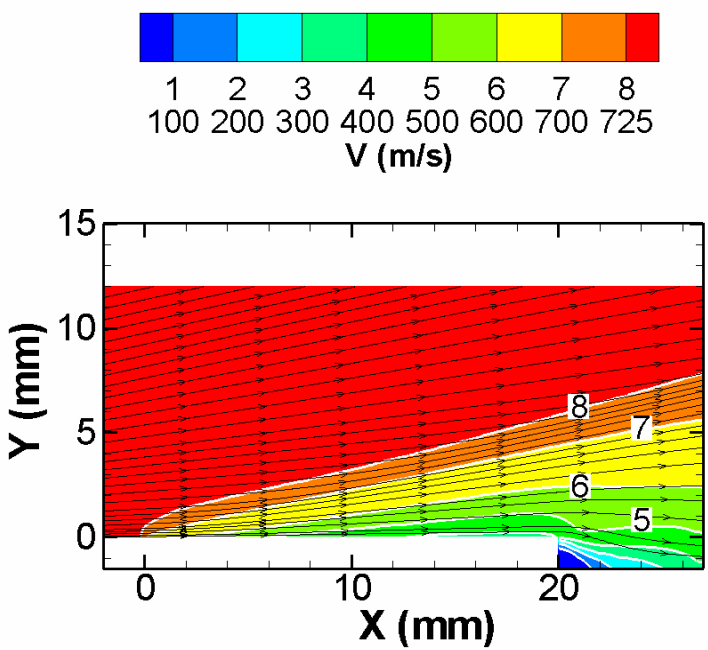

(a) $a_{M}=0.5$
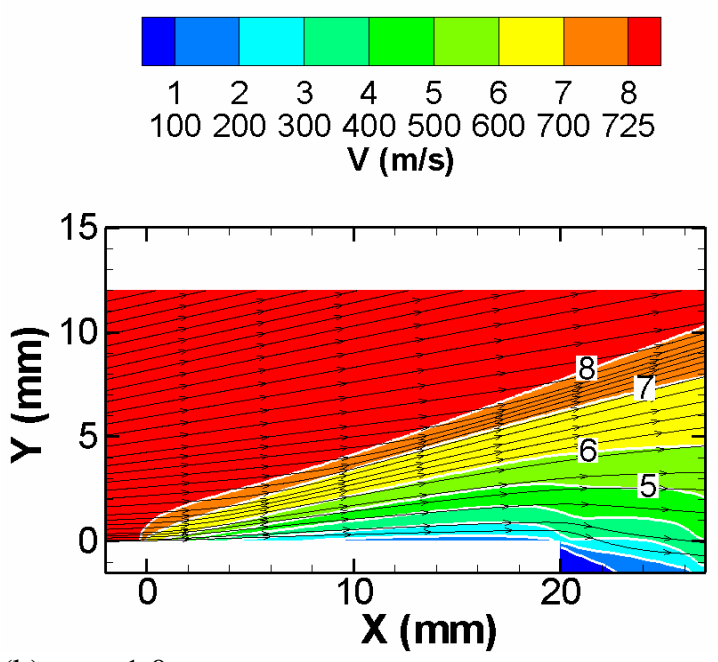

(b) $a_{M}=1.0$

Figure 2. Contour plots and streamlines of flow speed at two values of Maxwell's fraction

\section{Boundary Layer Velocity Profiles}

For each of the simulations, data are extracted along vertical slices corresponding to the locations where PLIF measurements were taken, $x=0,2.5,5,7.5,10,12.5,15,17.5$, and $20 \mathrm{~mm}$ from the leading edge. These data include $x$ and $y$ velocity components. Figures 3 (a) through (e) present some of the comparisons between the MONACO parametric results and the PLIF data. Various observations are made about the results.

Figure 3 (a) illustrates the measured velocity profiles at the leading edge of the flat plate. The fact that the simulations match the experimental data indicates that the correct inflow conditions are employed. Furthermore, all these figures indicate that the data agree in the freestream region above the boundary layer. However, the simulated $V_{x}$ profiles, at $x=20 \mathrm{~mm}$ and $y>\sim 6 \mathrm{~mm}$, shown in Fig. 3 (e), are near the right edge of the error bounds of the PLIF data. This is due to an adverse pressure gradient believed to be caused by windtunnel test flow phenomena, beyond the specified computational domain, and thus, not captured by the simulation. The adverse pressure gradient is more pronounce within the boundary layer.

The boundary layer is where the flow-field is affected by different gas-surface interaction models and different levels of gas-surface accommodation. When full gas-surface accommodation conditions are specified, by setting all accommodation coefficients equal to one, the Maxwell and CLL models yield the same boundary layer profiles, as expected from the definition of the models. In addition, the Maxwell and CLL models yield essentially the same results for $a_{M}=\sigma_{t}=0.5$ and 0.75 . However, the Maxwell and CLL models differ significantly at $a_{M}=\sigma_{t}=0$ and 
0.25. Hence, the Maxwell and CLL models are equivalent when $a_{M}=\sigma_{t} \geq 0.5$ for these flow conditions, while $a_{E_{i} \text { int }}$ $=\alpha_{n}=1.00$ in the CLL model.

The simulations involving full gas-surface accommodation, $a_{M}=\sigma_{t}=1.0$, give the overall best agreement with the measured data for the $V_{x}$ profiles, except at the transition from the boundary layer to the freestream, which includes the diffuse shock. The simulations with $a_{M}=\sigma_{t}=0.75$ provide the overall best agreement with the measured data for the $V_{y}$ profiles, and the $V_{x}$ profiles in the transition from the boundar layer to the freestream. Hence, the level of accommodation that provides the overall best agreement among both the $V_{x}$ and $V_{y}$ profiles is a compromise between 0.75 and 1.0. Simulations with the average accommodation, $a_{M}=\sigma_{t}=0.875$, result in profiles lying in the middle, in accord with the linear variation of the profiles with accommodation, when the accommodation level is greater than 0.5 . Hence, the overall best agreement, among the considered accommodation levels of the $a_{M}$ or $\sigma_{t}$ sensitivity studies, occurs when $a_{M}=\sigma_{t}=0.875$.
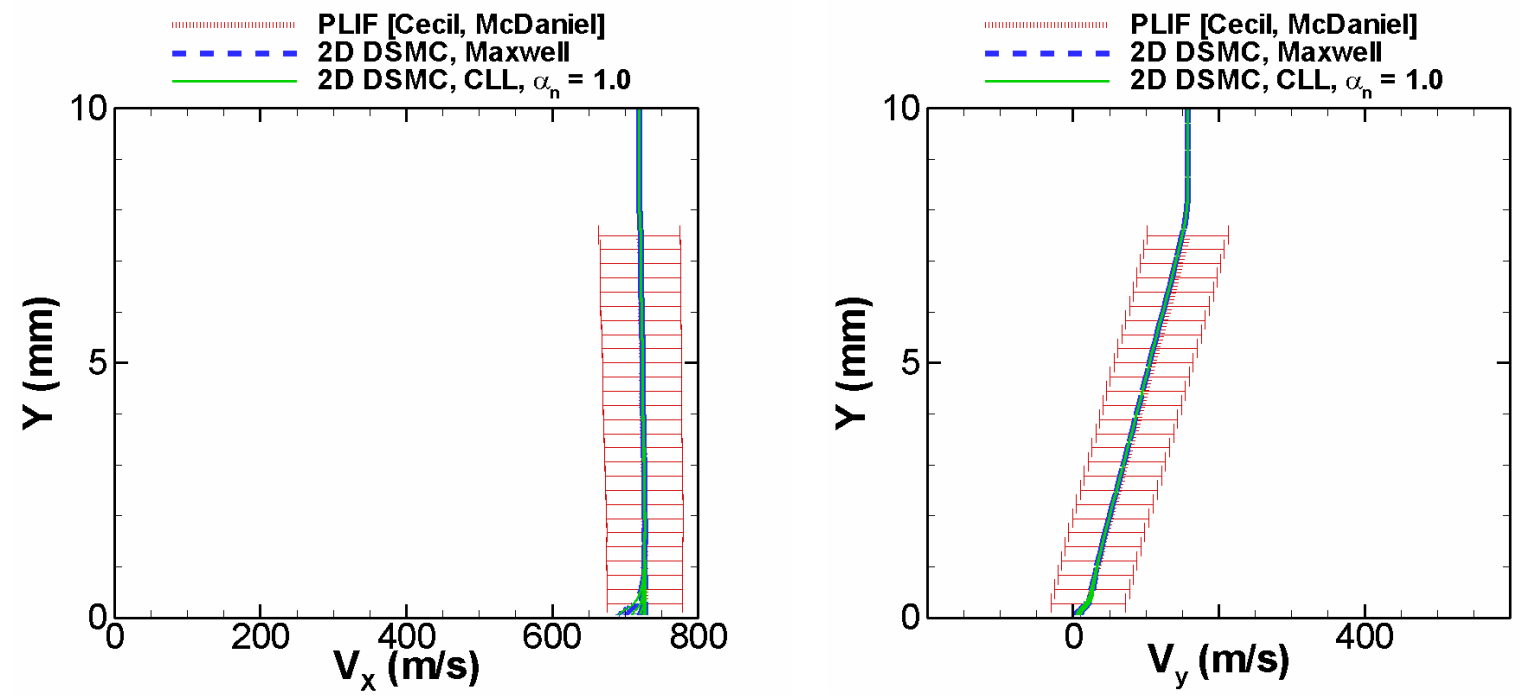

(a) Boundary layer velocity profiles at $x=0 \mathrm{~mm}$
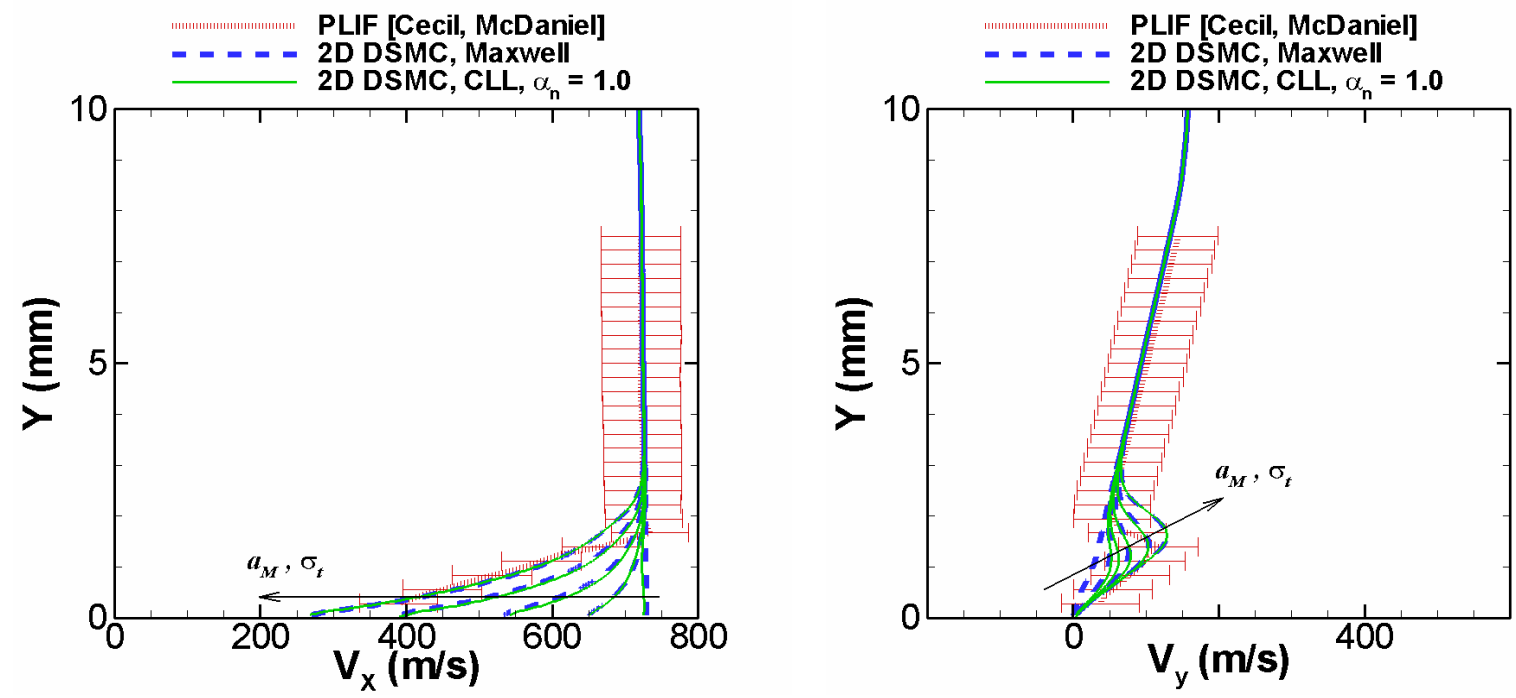

(b) Boundary layer velocity profiles at $x=5 \mathrm{~mm}$

Figure 3. Comparison of boundary layer velocity profiles between PLIF windtunnel tests and MONACO simulations with different gas-surface interaction models at various levels of accommodation 

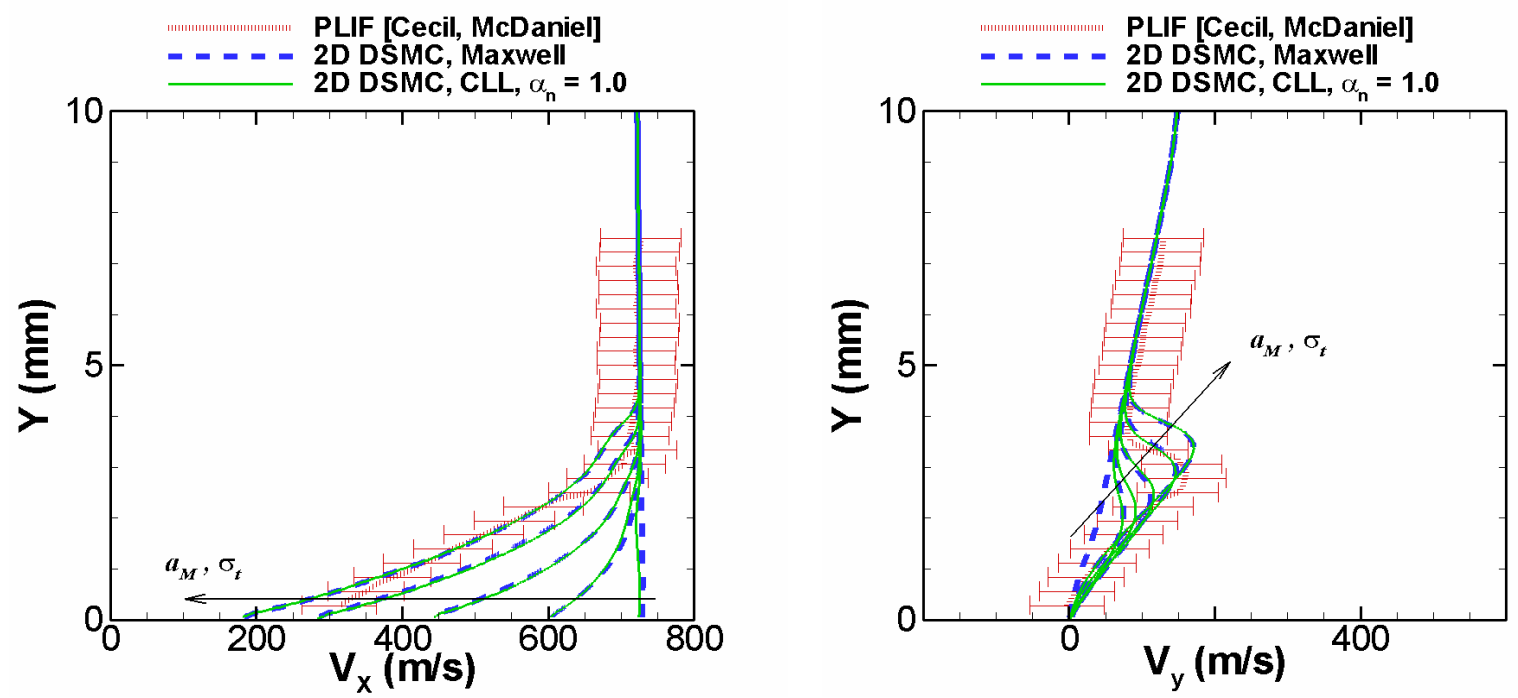

(c) Boundary layer velocity profiles at $x=10 \mathrm{~mm}$
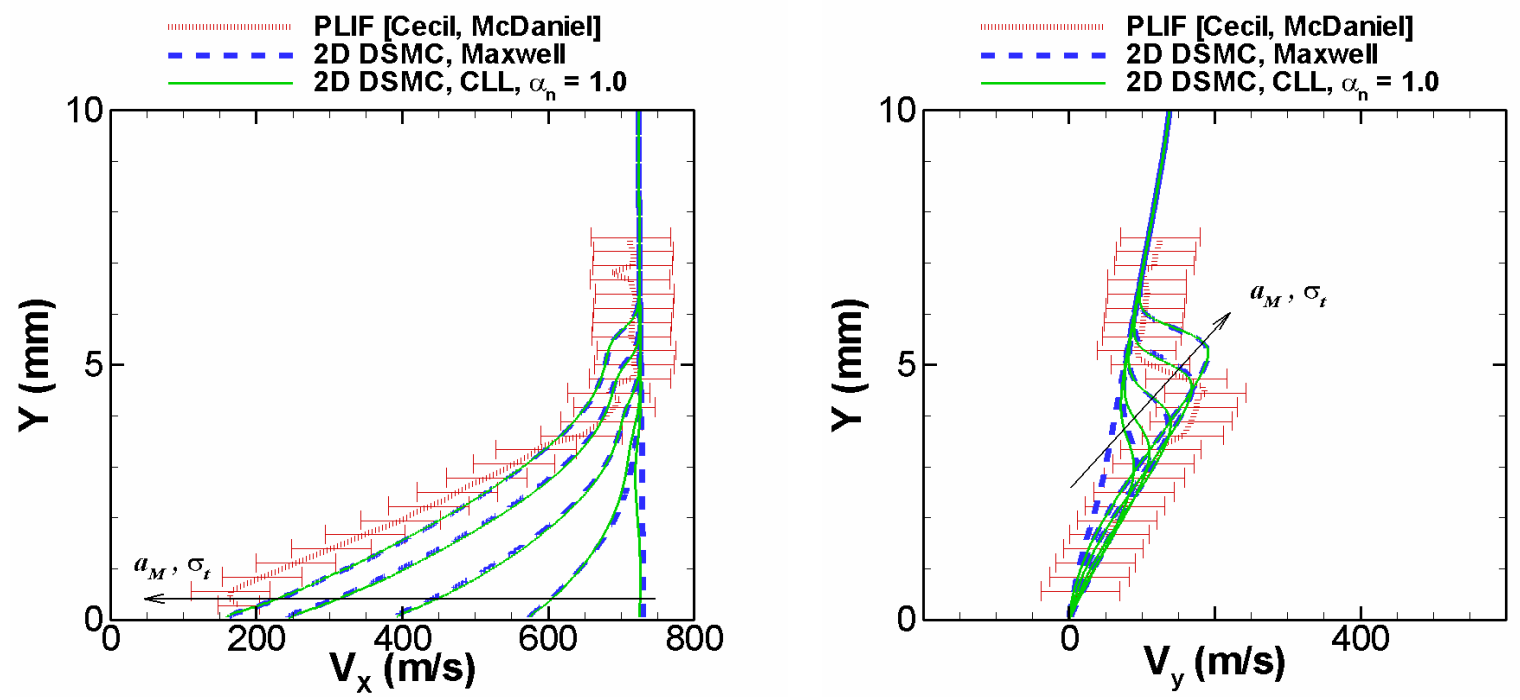

(d) Boundary layer velocity profiles at $x=15 \mathrm{~mm}$


(e) Boundary layer velocity profiles at $x=20 \mathrm{~mm}$

Figure 3. Concluded 
The sensitivity study of normal kinetic energy $\alpha_{n}$, confirms that $V_{x}$, the tangential component of velocity, is not significantly affected. The variation of $\alpha_{n}$ does affect $V_{y}$, the normal component of velocity, but not to the extent of the effects due to the same changes in $\sigma_{t}$. This study indicates that it is possible to improve the agreement in $V_{y}$, between the CLL simulation with $\sigma_{t}=1.0$ and the measured data, by significantly reducing $\alpha_{n}$; however, there is no indication in the experiments found in literature, or even in physical reasoning, that low normal kinetic energy accommodation would occur along with full tangential momentum accommodation $\sigma_{t}$. Hence, this case can not be favored over the $\sigma_{t}=0.875$ case.

\section{Spatial Variation of Molecular Velocity Distributions}

In the contour and boundary layer simulation plots, the flow velocity is the local mean molecular velocity, of the velocity distribution of the simulation molecules. The PLIF boundary layer plots also give the local mean molecular velocity. The mean molecular velocity is an accurate representation of a bulk velocity in translational equilibrium. A system of molecules in translational equilibrium has each of their velocity components distributed in a Gaussian or normal distribution. A set of normal velocity component distributions is a Maxwellian velocity distribution. For rarefied transitional flows, there may be regions with translational nonequilibrium. A system of molecules in translational nonequilibrium has at least one of their velocity components distributed in a non-normal distribution, e.g., a distribution with multiple peaks or with a skewed shape. Thus, the mean molecular velocity may not be an accurate representation of the bulk velocity in translational nonequilibrium. The degree to which the velocity distribution is Maxwellian, indicates the utility of employing the mean velocity to represent the bulk velocity of the local system of gas molecules. Knowledge of where the mean velocity is an inaccurate representation due to translational nonequilibrium enables a more accurate interpretation of the corresponding flow conditions. Hence, molecular velocity distributions are examined at specified points. Consideration is given to nonequilibrium in the boundary layer near the flat plate surface with full gas-surface accommodation. Full gas-surface accommodation yields the greatest amount of translational nonequilibrium because it incurs the greatest changes in normal and tangential kinetic energy.

Table 4 presents statistics of molecular velocity probability distributions at selected computational cells along the $x$ direction and next to the flat plate at $y=0 \mathrm{~mm}$. These cells have their centers at a height of $y=0.06 \mathrm{~mm}$ from the surface. The statistics have various trends associated with increasing $x$ position. The number of samples lowers with $x$ because of lower density, leaving fewer simulation molecules to sample. The variation of percentage difference, between the mean and most probable values, suddenly increases at $x=2.5 \mathrm{~mm}$, and then subsequently lowers with $x$. This percentage difference indicates the degree of translational nonequilibrium, which in this case is due to the initial wave of rebounding molecules from the flat plate surface. At equilibrium, the percentage difference is zero by definition. The cause of the tendency towards equilibrium, after the initial disturbance of reflected molecules, is attributed to more evenly balanced forward and backward scatter from adjacent cells, at positions further into the interior of the surface. The larger percentage difference and standard deviation of the $\xi_{y}$ distributions is attributed to the obvious more significant change in the normal component of momentum due to collision with the surface. The standard deviation, which is indicative of the distribution spread, does not necessarily vary directly with percentage difference or the degree of translational nonequilibrium: whereas, direct variation is found for the $\xi_{x}$ distributions; inverse variation is found for the $\xi_{y}$ distributions, between $x=2.5$ and $20 \mathrm{~mm}$.

Table 4. 2D DSMC statistics of molecular velocity distributions at $y=0 \mathbf{m m}$ for various values of $x$

\begin{tabular}{|c|c|c|c|c|c|c|c|c|c|}
\hline$x(\mathrm{~mm})$ & $\begin{array}{l}\text { No. of } \\
\text { Samples }\end{array}$ & $\begin{array}{c}\xi_{x} \\
\text { Std. Dev. }\end{array}$ & Mean & M. P. & $\% \operatorname{Diff}^{\dagger}$ & $\begin{array}{c}\xi_{y} \\
\text { Std. Dev. }\end{array}$ & Mean & M. P. & $\% \operatorname{Diff}^{\dagger}$ \\
\hline 0 & $6,441,210$ & 217.8 & 636.0 & 747.0 & 16.1 & 109.2 & 636.0 & 9.6 & 52.3 \\
\hline 2.5 & $4,387,893$ & 335.7 & 403.4 & 705.2 & 54.5 & 204.3 & 403.4 & -35.5 & 290.9 \\
\hline 5.0 & $3,299,356$ & 333.2 & 251.2 & 394.0 & 44.3 & 234.6 & 251.2 & -69.3 & 220.9 \\
\hline 7.5 & $2,752,768$ & 326.8 & 201.1 & 308.3 & 42.1 & 256.2 & 201.1 & -109.0 & 205.0 \\
\hline 20 & $1,255,890$ & 299.3 & 217.1 & 258.7 & 17.5 & 273.3 & 217.1 & -147.5 & 182.9 \\
\hline
\end{tabular}

most probable value

$\dagger$ percentage difference magnitude between mean and most probable value

Figure 4 illustrates the normalized probability distributions, corresponding to Table 4 . At the computational cell adjacent to the leading edge, $x=0 \mathrm{~mm}$, both the $\xi_{x}$ and $\xi_{y}$ distributions are in near equilibrium. Here, they are not significantly affected by surface reflected molecules because the cell length is only approximately equal to the local mean-free-path. The degree of nonequilibrium described by the distribution shape agrees with the trend in percentage difference between the mean and most probable values. At $x=2.5 \mathrm{~mm}$, the distributions are significantly 
skewed. At $x=5.0 \mathrm{~mm}$, the $\xi_{x}$ distribution are only slightly skewed. By $x=7.5 \mathrm{~mm}$, the $\xi_{x}$ distributions have returned to a near normal shape. Molecules traversing this distance will have encountered an average of about 21 collisions, according to the computed local mean-free-path values. In contrast with the $\xi_{x}$ distributions, the $\xi_{y}$ distributions remain in translational nonequilibrium along the entire length of the flat plate.
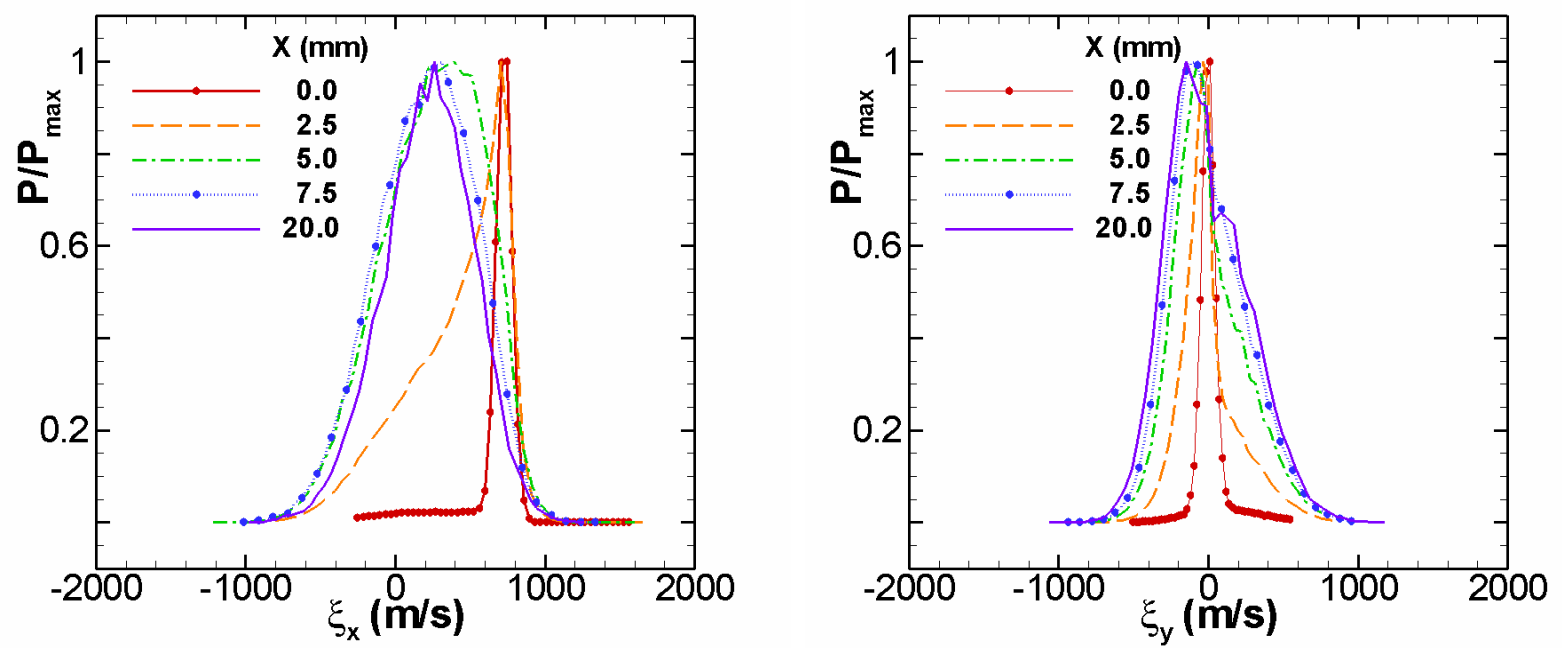

Figure 4. 2D DSMC spatial variation along $x$ at the surface, $y=0 \mathrm{~mm}$, of molecular velocity probability distributions within boundary for full gas-surface accommodation

Figures 5 (a) and (b) present the evolution of molecular velocity distributions with $y$, at two positions along the plate, $x=2.5$ and $20 \mathrm{~mm}$, respectively. To reduce clutter only three of the examined distributions are plotted. At $x=$ $2.5 \mathrm{~mm}$, both the $\xi_{x}$ and $\xi_{y}$ distributions evolve toward equilibrium as $y$ increases. The rate of this evolution is greater than that of the $\xi_{x}$ distributions with $x$ along the surface, given the smaller increments in $y$, c.f. Fig. 4 . This is expected because the presence of molecules reflected directly from the surface decreases with vertical distance from the surface. Table 5 (a) below provides the corresponding statistics. Again, the number of samples reflects the gas density, and the percentage difference between the mean and most probable values reflects the level of translational nonequilibrium. Smaller values of percentage difference occur at $y \geq 1.0 \mathrm{~mm}$, where the distributions are near normal, for both the $\xi_{x}$ and $\xi_{y}$ distributions. Molecules diffusing upward from the surface encounter an average of 5 collisions before reaching $y=1.0 \mathrm{~mm}$ at $x=2.5 \mathrm{~mm}$.
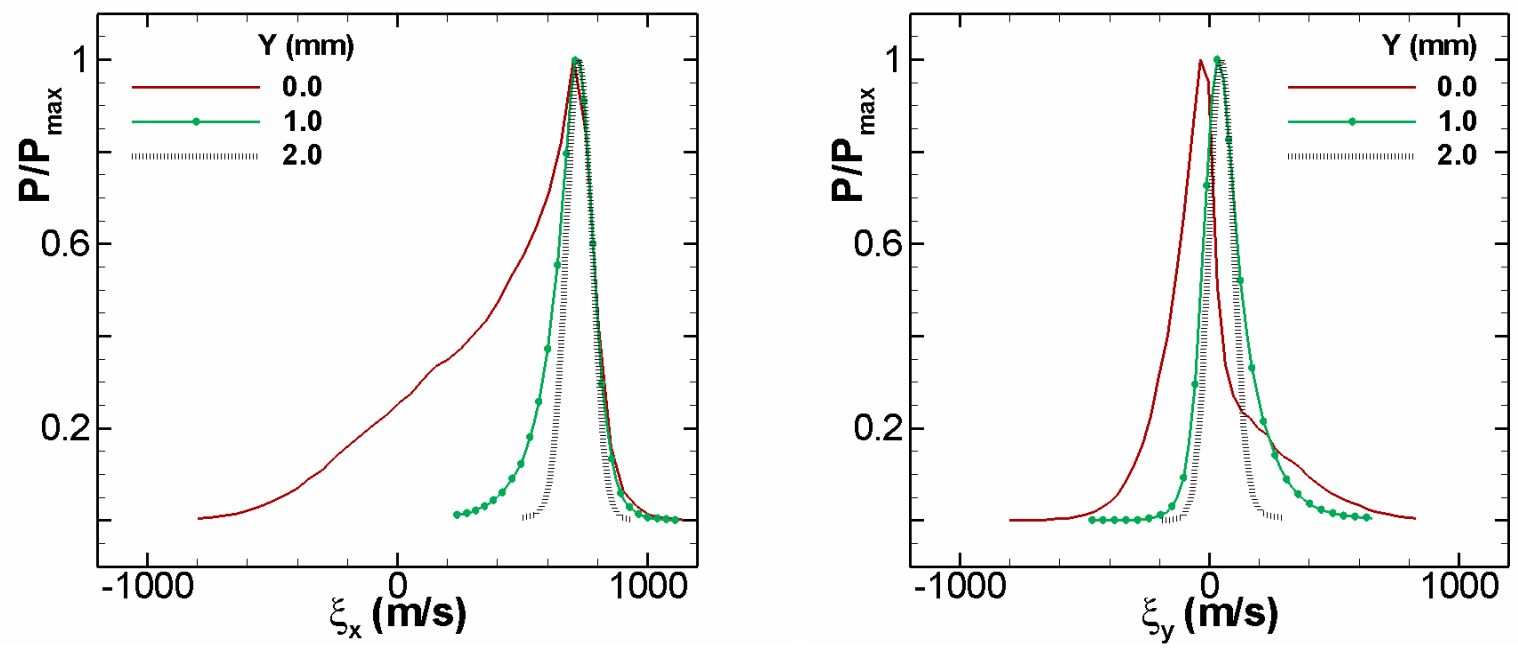

(a) Simulated molecular velocity distribtutions of full accommodation at $x=2.5 \mathrm{~mm}$

Figure 5. 2D DSMC spatial variation along $y$ of molecular velocity probability distributions within boundary layer for full gas-surface accommodation 

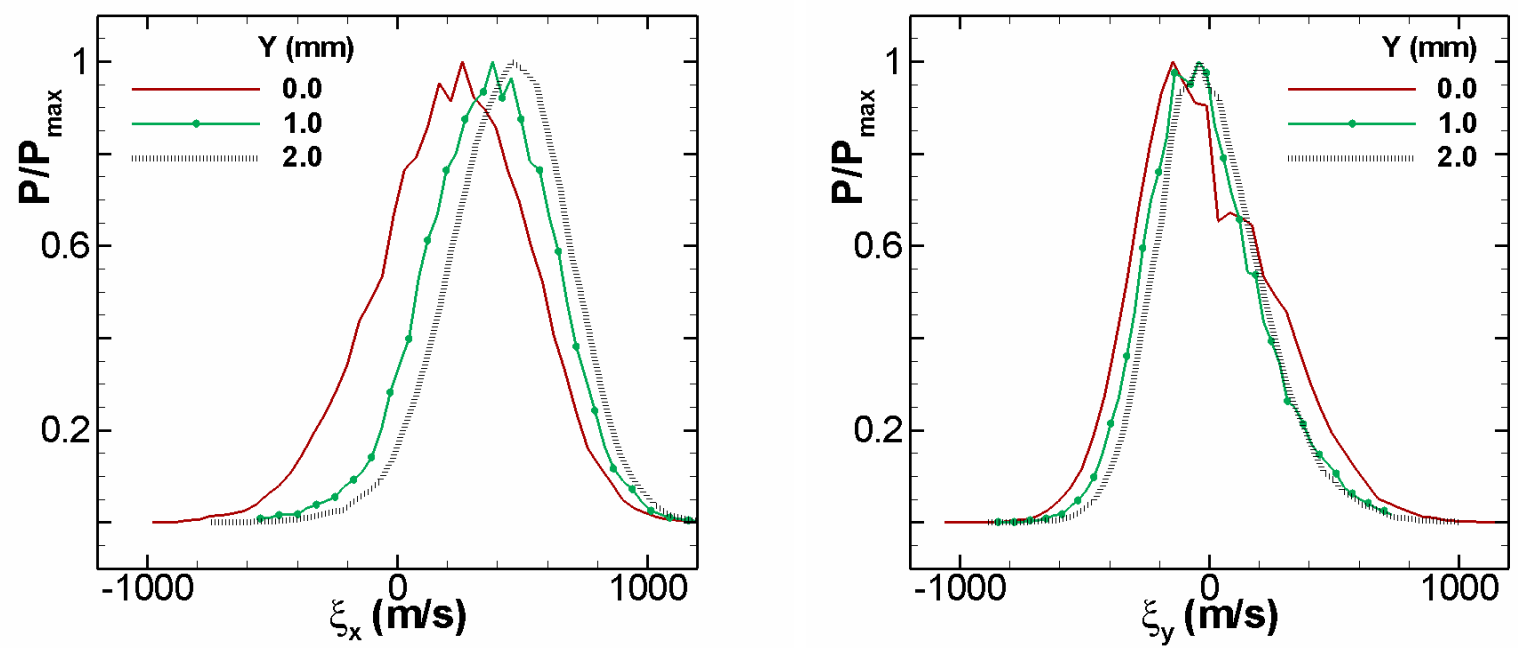

(b) Simulated molecular velocity distribtutions of full accommodation at $x=20 \mathrm{~mm}$

Figure 5. Concluded

At $x=20 \mathrm{~mm}$ and $y=0 \mathrm{~mm}$, the $\xi_{x}$ distribution is already near equilibrium. As $y$ increases the $\xi_{x}$ distribution remains near normal, as shown in Fig. 5 (b) and Table 5 (b). In contrast, at $x=20 \mathrm{~mm}$ and $y=0 \mathrm{~mm}$, the $\xi_{y}$ distribution is skewed and has two peaks. At $y=0.3 \mathrm{~mm}$, the skew is slightly reduced and the second peak disappears. By $y=1.0 \mathrm{~mm}$, the $\xi_{y}$ distributions are only slightly skewed, but has two peaks again. From $y=0.5$ to $2.0 \mathrm{~mm}$, the percentage difference between the mean and most probable values indicates an increase in nonequilibrium for the $\xi_{y}$ distribution with $y$. This can be explained by the backward facing step, at $x=20 \mathrm{~mm}$, corresponding to the end of the flat plate model and shown in Fig. 2 above.

Table 5. 2D DSMC statistics of molecular velocity distributions at $x=2.5$ and $20 \mathrm{~mm}$ for various values of $\mathbf{y}$

\begin{tabular}{|c|c|c|c|c|c|c|c|c|c|}
\hline$y(\mathrm{~mm})$ & $\begin{array}{l}\text { No. of } \\
\text { Samples }\end{array}$ & $\begin{array}{c}\xi_{x} \\
\text { Std. Dev. }\end{array}$ & Mean & M. P. & $\% \operatorname{Diff}^{\dagger}$ & $\begin{array}{c}\xi_{y} \\
\text { Std. Dev. }\end{array}$ & Mean & M. P.* & $\%$ Diff \\
\hline \multicolumn{10}{|c|}{ (a) $x=2.5 \mathrm{~mm}$} \\
\hline 0 & $4,387,893$ & 335.7 & 403.4 & 705.2 & 54.5 & 204.3 & 6.6 & -35.5 & 290.1 \\
\hline 0.3 & $6,540,059$ & 250.3 & 540.9 & 713.9 & 27.6 & 171.2 & 40.9 & 2.4 & 177.7 \\
\hline 0.5 & $8,533,708$ & 189.4 & 599.6 & 706.2 & 16.3 & 148.4 & 69.9 & 21.0 & 107.7 \\
\hline 1.0 & $9,800,428$ & 118.6 & 668.7 & 729.7 & 8.7 & 116.7 & 81.6 & 33.7 & 82.9 \\
\hline 2.0 & $7,946,681$ & 57.8 & 733.5 & 730.8 & 1.1 & 59.7 & 52.0 & 48.4 & 7.2 \\
\hline \multicolumn{10}{|c|}{ (b) $x=20 \mathrm{~mm}$} \\
\hline 0 & $1,255,890$ & 299.3 & 217.1 & 258.7 & 17.5 & 273.3 & -6.6 & -147.5 & 182.9 \\
\hline 0.3 & $1,304,345$ & 274.8 & 281.5 & 228.8 & 20.7 & 260.8 & -20.8 & -108.7 & 135.8 \\
\hline 0.5 & $1,346,391$ & 269.3 & 311.0 & 319.4 & 2.7 & 250.8 & -23.6 & -67.5 & 93.4 \\
\hline 1.0 & $1,385,069$ & 255.9 & 365.5 & 382.2 & 4.5 & 234.2 & -14.4 & -4.2 & 110.3 \\
\hline 2.0 & $1,741,867$ & 237.9 & 444.8 & 457.5 & 2.8 & 215.6 & 12.5 & -39.4 & 385.6 \\
\hline
\end{tabular}

most probable value

$\dagger$ percentage difference magnitude between mean and most probable value

In summary, translational nonequilibrium is found very near the flat plat surface due to reflected molecules. Within significant translational nonequilibrium, the most probable value represents the bulk velocity better than the mean value. Nonequilibrium $\xi_{x}$ distributions occur at $x \leq 7.5 \mathrm{~mm}, 21$ mean-free-paths past the leading edge, and $y<$ $1.0 \mathrm{~mm}, 5$ mean-free-paths above the surface. Nonequilibrium $\xi_{y}$ distributions occur throughout the entire flat plate surface at less than $y=1.5 \mathrm{~mm}$, which is 5 mean-free-paths above the surface at $x=2.5 \mathrm{~mm}, 10$ mean-free-paths past the leading edge; at $x>2.5 \mathrm{~mm}, y=1.5 \mathrm{~mm}$ is fewer than 5 mean-free-paths. Nevertheless, evolution toward translational equilibrium is observed as $x$ and $y$ increase, with the rate of evolution toward translational equilibrium being significantly greater in the $y$ direction. 


\section{Effects of Gas-Surface Accommodation on Molecular Distributions}

Molecular distribution plots also provide a detailed perspective of the effects of different gas-surface interaction models and of varying the gas-surface accommodation. Molecular velocity distributions at two locations over the flat plate surface are examined. The molecular velocity distributions at $x=0 \mathrm{~mm}$ are unaffected by changes in gassurface interaction model or gas-surface accommodation. These distributions are shown above in Fig. 4 at $x=0 \mathrm{~mm}$. These distributions correspond to the first computational cell over the flat plate surface, which begins at $x=0 \mathrm{~mm}$ and ends at $x=0.25 \mathrm{~mm}$. Molecular velocity distributions at $x=5.0 \mathrm{~mm}$ are shown below in Fig. 6 . This figure illustrates that the degree of translational nonequilibrium next to the surface is proportional to the level of gassurface accommodation. It also shows that the Maxwell and CLL models give identical results only for full gassurface accommodation, $a_{M}=\sigma_{t}=1.0$. At partial levels of gas-surface accommodation, the models yield similar $\xi_{x}$ distributions. However, the $\xi_{y}$ distributions, at $\xi_{y}>0$, are significantly different.
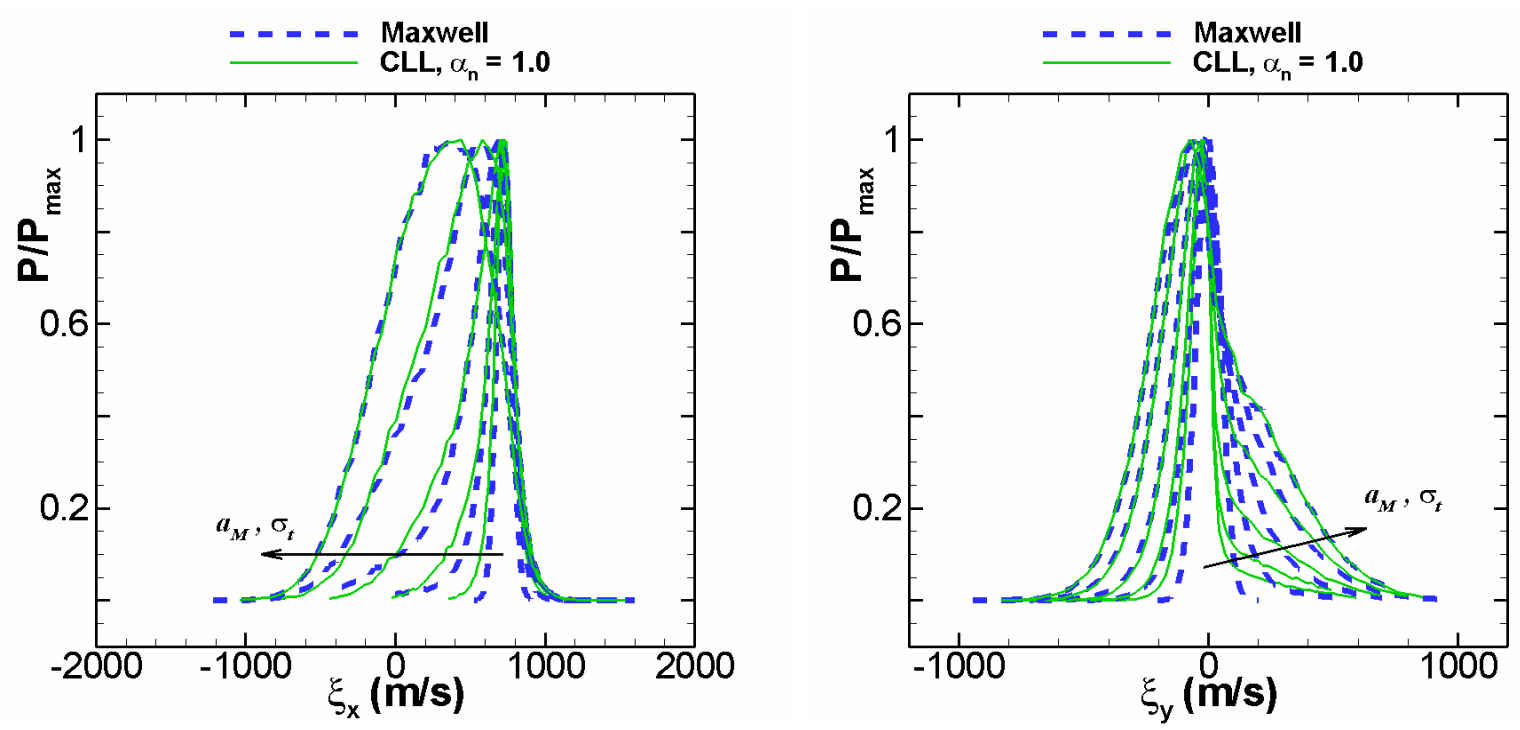

Figure 6. 2D DSMC molecular velocity probability distributions of computational cell containing point $(x, y)$ $=(5.0,0.0) \mathrm{mm}$, of two gas-surface interaction models at various levels of gas-surface accommodation: $a_{M}, \sigma_{t}=0,0.25,0.5,0.75$ and 1.0

The molecular surface scattering plots are also examined at the two positions $x=0$ and $5.0 \mathrm{~mm}$ over the flat plate surface. These are probability distribution plots of gas-surface reflection or scattering angle, the angle between the surface horizon and the molecular velocity upon departure from the surface. Figures 7 (a) and (b) present the distributions. The scattering plots show the essential difference between the Maxwell and CLL gas-surface interaction model. The Maxwell scattering distributions have unrealistic peaks due to the specular angle at partial levels of accommodation. The CLL scattering distributions are petal-shaped, similar to observations of reflected rarefied molecular beams from clean flat surfaces. ${ }^{8,9}$ As required, the scatter plots further confirm that both models are equivalent at full accommodation. There, they yield the Lambert or cosine distribution of optics theory, which applies to the random distribution of gas-surface scattering angles. The scattering plots also show variations due to changing accommodation and $x$ position along the flat plate surface. The Maxwell distributions have abrupt changes with increasing accommodation and position; in contrast, the CLL distributions vary smoothly with accommodation and position, with only a slight changes due to position. 

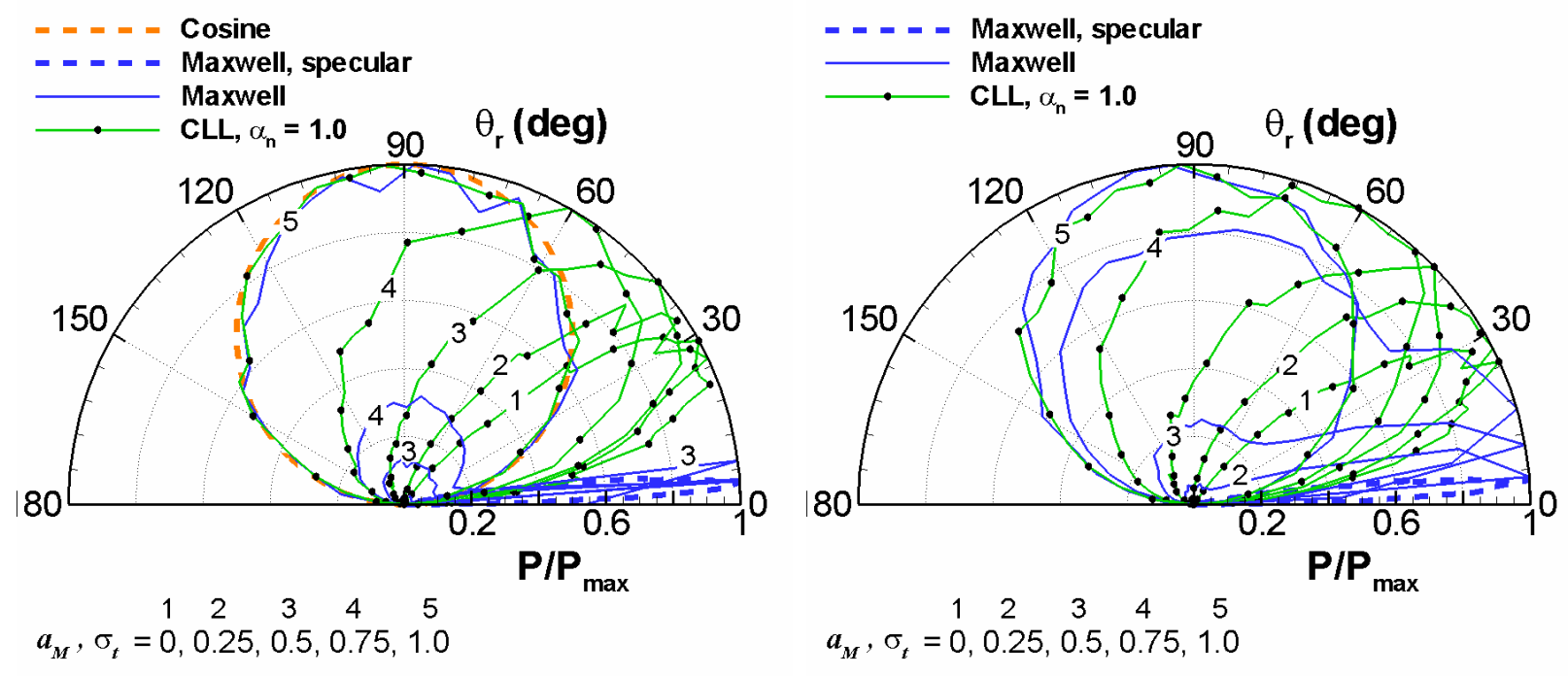

(a) $x=0 \mathrm{~mm}$

(b) $x=5.0 \mathrm{~mm}$

Figure 7. 2D DSMC probability distributions of reflected molecular velocity angle of two flat plate surface faces, $y=0 \mathrm{~mm}$, of two gas-surface interaction models at various levels of gas-surface accommodation

\section{Conclusion}

The Maxwell and CLL gas-surface interaction models, commonly used in DSMC, were reviewed. Their implementation into the MONACO DSMC code was described. To examine the gas-surface interaction models, computational simulations were made of an existing windtunnel test study, performed by Cecil and McDaniel, involving a free jet expansion of nitrogen into rarefied hypersonic flow over a flat plate. They measured the windtunnel test flow field properties, near the flat plate, with planar laser induced fluorescence (PLIF) of seeded iodine, within a nitrogen flow. A detailed study was made of the flat plate near-field flow condition, with MONACO simulations employing a nonuniform inflow condition, with data taken from the PLIF measurements, and assuming pure nitrogen.

The first part of the study involved examining the effects of varying gas-surface accommodation, for each gassurface interaction model, on the boundary layer velocity. The computed set of profiles encompassed the PLIF data, except for $x>12.5 \mathrm{~mm}$, where an adverse pressure gradient resided due to windtunnel test flow phenomena outside the specified computational domain. The Maxwell and CLL models gave the same boundary layer profiles at 50 to $100 \%$ gas-surface accommodation, based on Maxwell's fraction and CLL's tangential momentum accommodation coefficient. In addition, 87.5\% gas-surface accommodation yielded the overall best agreement between the simulations and the PLIF data.

The second part of the study involved examining molecular velocity distributions at specified locations in the flow-field. An examination of the spatial variation of the distributions revealed translational nonequilibrium within 5 local mean-free-paths above the flat plate surface due to reflected molecules. However, evolution toward translational equilibrium was observed as $x$ and $y$ increase, with the rate of evolution toward translational equilibrium being significantly greater in the $y$ direction. Within the region of significant translational nonequilibrium, the distributions were better characterized by the most probable value, rather than the mean value. An examination was then made of the effects of different gas-surface interaction models and of varying the gassurface accommodation on the distributions. The Maxwell and CLL gas-surface interaction models gave similar $\xi_{x}$ distributions, but distinct $\xi_{y}$ distributions, at partial levels of gas-surface accommodation. In addition scattering distributions were examined. The Maxwell model resulted in distributions with unrealistic peaks due to specular reflection; whereas, the CLL model resulted in petal-shaped distributions, similar to observations of molecular beam studies, reported in the literature. Moreover, while the Maxwell scattering distributions experienced abrupt changes with increasing accommodation and position, the CLL distributions varied smoothly.

In conclusion, the study provided a detailed understanding of the effects of gas-surface interactions on the velocity field and molecular velocity distributions within the boundary layer, near the solid surface. 2D DSMC 
simulations provided good agreement with PLIF windtunnel test boundary layer velocity profiles, using a proper specification of gas-surface accommodation, with either the Maxwell or the CLL gas-surface interaction model; however, the CLL model is physically more realistic. Suggestions for future work related to this study are: the extension of the models to three-dimensional simulations, the examination of energy distributions, the examination of the effects of wall temperature, and the examination of the effects of surface material properties.

\section{Acknowledgements}

This work is sponsored by the Space Vehicle Technology Institute, under NASA grant NCC-3989 with joint sponsorship from the Department of Defense. The authors gratefully acknowledge windtunnel data provided by Eric Cecil and Prof. James McDaniel from the University of Virginia.

\section{References}

${ }^{1}$ Schaaf, S. A., and Chambré, P. L., Flow of Rarefied Gases, Princeton University Press, Princeton, New Jersey, 1961.

${ }^{2}$ Gombosi, T. I., Gaskinetic Theory, Cambridge University Press, Cambridge, England, 1994.

${ }^{3}$ Cercignani, C., "Scattering Kernels for Gas-Surface Interactions," Transport Theory and Statistical Physics, Vol. 2, No. 1, 1972, pp. 27-53.

${ }^{4}$ Wenaas, E. P., "Equilibrium Cosine Law and Scattering Symmetry at the Gas-Surface Interface," Jounal of Chemical Physics, Vol. 54, No. 1, 1971, pp. 376-388.

${ }^{5}$ Kuscer, I., "Reciprocity in Scattering of Gas Molecules by Surfaces," Surface Science, Vol 25, 1971, pp. $225-237$.

${ }^{6}$ Maxwell, J. C., "On Stresses in Rarified Gases Arising from Inequalities of Temperature," Philosophical Transactions of the Royal Society of London, Vol. 170, 1879, pp. 231-256.

${ }^{7}$ Cercignani, C., "Boundary Value Problems in Linearized Kinetic Theory," SIAM-AMS Proceedings, edited by Birkhoff, G., et al, Vol. 1, American Mathematical Society, Providence, Rhode Island, 1968, pp. 249-268.

${ }^{8}$ Schaaf, S. A., "Mechanics of Rarefied Gases," Encyclopedia of Physics: Fluid Dynamics II, edited by S. Flügge and C. Truesdell, Vol. 8, Springer-Verlag, Berlin, 1963, pp. 591-624.

${ }^{9}$ Hinchen, J. J., and Foley, W. M., "Scattering of Molecular Beams by Metallic Surfaces," ${ }^{\text {th }}$ International Symposium on Rarefied Gas Dynamics, edited by J. H. de Leeuw, Vol. 2, Academic Press, New York, 1966, pp. 505-517.

${ }^{10}$ Schamberg, R., "A New Analytic Representation of Surface Interaction for Hyperthermal Free-Molecule Flow with Application to Satellite Drag," Proceedings of the Heat Transfer and Fluid Mechanics Institute, Stanford University Press, 1959, pp. 1-14.

${ }^{11}$ Nocilla, S., "The Surface Re-Emission Law in Free Molecule Flow," $3^{\text {rd }}$ International Symposium on Rarefied Gas Dynamics, edited by J. A. Laurmann, Academic Press, New York, 1963, pp. 327-346.

${ }^{12}$ Hurlbut, F. C., and Sherman, F. S., "Application of the Nocilla Wall Reflection Model to Free-Molecule Kinetic Theory," The Physics of Fluids, Vol. 11, No. 3, 1968, pp. 486-496.

${ }^{13}$ Cercignani, C. and Lampis, M., "Kinetic Models for Gas-Surface Interaction," Transport Theory and Statistical Physics, Vol. 1, No. 2, 1971, pp. 101-114.

${ }^{14}$ Agbormbai, A. A., "A Statistical Theory for Gas Surface Interactions in Rarefied Hypersonic Flows," Imperial College of Science and Technology, I.C. Aero Report 88-01, 1988.

${ }^{15}$ Cercignani, C., "Scattering Kernels for Gas-Surface Interaction," Hypersonic Flows for Reentry Problems, edited by J.-A. Désidéri, R. Glowinski and J. Périaux, Springer-Verlag, New York, 1990, pp. 35-54.

${ }^{16}$ Cercignani, C., and Lampis, M., "New Scattering Kernel for Gas-Surface Interaction,” AIAA Journal, Vol. 35, No. 6, 1997, pp. 1000-1011.

${ }^{17}$ Lord, R. G., "Application of the Cercignani-Lampis Scattering Kernel to Direct Simulation Monte Carlo Calculations," $17^{\text {th }}$ International Symposium on Rarefied Gas Dynamics, edited by A. E. Beylich, Aachen, VCH, Weinheim, Germany, 1990, pp. 1427-1433.

${ }^{18}$ Bird, G. A., Molecular Gas Dynamics and the Direct Simulation of Gas Flows, Oxford University Press, 1994.

${ }^{19}$ Dietrich, S., and Boyd, I. D., "Scalar and Parallel Optimized Implementation of the Direct Simulation Monte Carlo Method," Journal of Computational Physics, Vol. 126, 1996, pp. 328-342.

${ }^{20}$ Bird, G. A., "Monte Carlo Simulation in an Engineering Context," $12^{\text {th }}$ International Symposium on Rarefied Gas Dynamics, edited by S. S. Fisher, AIAA, New York, 1981, pp.

${ }^{21}$ Koura, K., and Matsumoto, H., "Variable Soft Sphere Molecular Model for Air Species," Physics of Fluids, Vol. 4, 1992, pp. 1083-1085.

${ }^{22}$ Boyd, I. D., “Analysis of Rotational Nonequilibrium in Standing Shock Waves of Nitrogen,” AIAA Journal, Vol. 28, No. 11, 1990, pp. 1997-1999.

${ }^{23}$ Vijayakumar, P., Sun, Q., and Boyd, I. D., "Detailed Models of Vibrational-Translational Energy Exchange for the Direct Simulation Monte Carlo Method," Physics of Fluids, Vol. 11, 1999, pp. 2117-2126.

${ }^{24}$ Cecil, E. and McDaniel, J. C., "Planar Velocity and Temperature Measurements in Rarefied Hypersonic Flow Using Iodine LIF," $38^{\text {th }}$ AIAA Thermophysics Conference, AIAA Paper 2005-4695.

${ }^{25}$ HyperMesh, Version 7.0, Altair Engineering Inc., Troy, MI, 2004. 